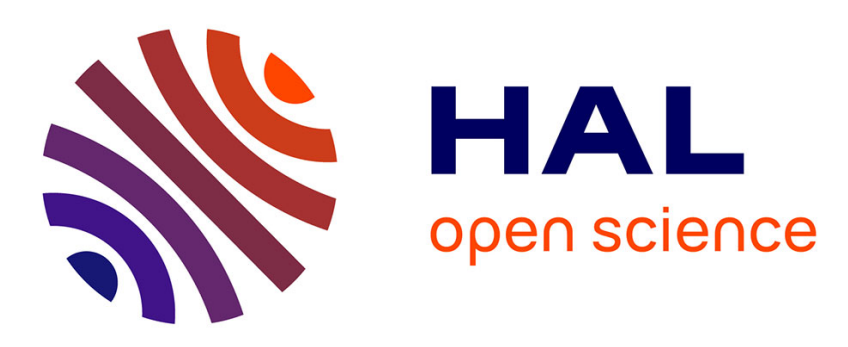

\title{
Un projet collectif de recherche autour du fonds Arthur Stieber
}

Cécile Courtaud, Juliette Rémy, Isabelle Lesueur, Bernadette Schnitzler, Soline Morinière, Marie Stahl, Georges Triantafillidis

\section{To cite this version:}

Cécile Courtaud, Juliette Rémy, Isabelle Lesueur, Bernadette Schnitzler, Soline Morinière, et al.. Un projet collectif de recherche autour du fonds Arthur Stieber. Archimède: archéologie et histoire ancienne, 2015, 2, pp.66-77. halshs-01587296

\section{HAL Id: halshs-01587296 \\ https://shs.hal.science/halshs-01587296}

Submitted on 14 Sep 2017

HAL is a multi-disciplinary open access archive for the deposit and dissemination of scientific research documents, whether they are published or not. The documents may come from teaching and research institutions in France or abroad, or from public or private research centers.
L'archive ouverte pluridisciplinaire HAL, est destinée au dépôt et à la diffusion de documents scientifiques de niveau recherche, publiés ou non, émanant des établissements d'enseignement et de recherche français ou étrangers, des laboratoires publics ou privés. 


\section{ARCHIMĖDE N $N^{\circ} 2$}

\section{DOSSIER THÉMATIQUE : ARCHIVES DE L'ARCHÉOLOGIE}

1 Marie STAHL

Introduction : la mémoire retrouvée des archéologues

9 Marie STAHL, Lucile SCHIRR

Les archives de I'archéologie : définition, législation, état des lieux

20 Dominique BEYER, Marie STAHL (collab. Catherine DUVETTE, Isabelle WEYGAND, Françoise LAROCHE-TRAUNECKER, Marie-José MORANT, Philippe QUENET) Les archives de la composante d'archéologie orientale de I'UMR 7044

41 Cassandre HARTENSTEIN

Le fonds Montet et la statue «maussade » de Ramsès II au Palais universitaire de Strasbourg

51 Soline MORINIÈRE

Les archives de I'archéologie au SRA Alsace : état des lieux et des fonds

59 Anne ROHFRITSCH

Les archives dites « manuscrites » des membres de l'École française d'Athènes :

l'exemple des «Strasbourgeois » (1846-1960)

66 Cécile COURTAUD, Isabelle LESUEUR, Soline MORINIĖRE, Juliette RÉMY,

Bernadette SCHNITZLER, Marie STAHL, Georges TRIANTAFILLIDIS

Un projet collectif de recherche autour du fonds Arthur Stieber

78 Soline MORINIÈRE

La gypsothèque de I'Université de Strasbourg : quand les statues parlent d'elles-mêmes

\section{LA CHRONIQUE D'ARCHIMÈDE}

94 Frédéric COLIN (éd.)

La Chronique d'Archimède. Bilan des activités scientifiques 2014-2015 de I'unité mixte de recherche 7044

\section{VARIA}

134 Sarah DERMECH

Couleurs, éclat et brillance des crânes surmodelés : le cas du Néolithique Proche-oriental

150 Cinzia BEARZOT

La violence de l'État. La condamnation à mort sans jugement dans la Grèce ancienne

160 Doris MEYER

Jusqu'au dernier mot. Martyr, débat public et résistance dans la littérature de l'Antiquité tardive et à Byzance

170 Vincent PUECH

Les biens fonciers des élites sénatoriales à Constantinople et dans ses environs (451-641)

194 Clara MILLOT

Entre les enfants d'Hérodote et les enfants d'Adam Smith. Pour une approche économique des données archéologiques

Retrouvez tous les articles de la revue ARCHIMĖDE sur :

http://archimede.unistra.fr/revue-archimede/archimede-2-2015 


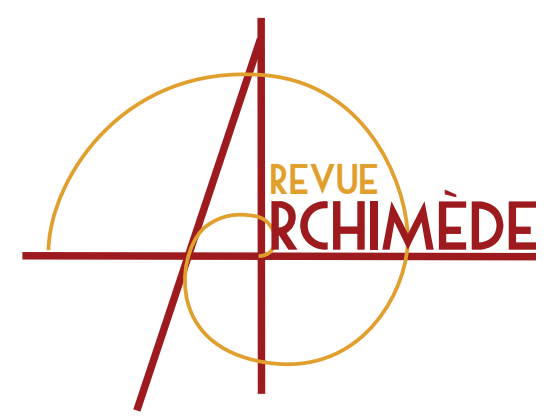

ARCHÉOLOGIE ET HISTOIRE ANCIENNE

\section{UN PROJET COLLECTIF DE RECHERCHE AUTOUR DU FONDS ARTHUR STIEBER}

\author{
Cécile COURTAUD \\ Chargée d'études documentaires, \\ DRAC Alsace \\ cecile.courtaud@culture.gouv.fr
}

\section{Juliette RÉMY}

Conservateur du patrimoine, Musées nationaux du palais de Compiègne

juliette.remy@culture.gouv.fr
Isabelle LESUEUR

Bibliothécaire-régisseur, Pôle d'archéologie

interdépartemental rhénan

isabelle.lesueur@pair-archeologie.fr

Bernadette SCHNITZLER

Conservateur en chef du patrimoine, Musée archéologique de Strasbourg

bernadette.schnitzler@strasbourg.eu
Soline MORINIÈRE

Chargée d'études documentaires, DRAC Alsace

soline.moriniere@culture.gouv.fr

Marie STAHL

Chargée d'études documentaires, DRAC Alsace, UMR 7044 Archimède

marie.stahl@culture.gouv.fr

\section{Georges TRIANTAFILLIDIS}

Ingénieur d'études, DRAC Alsace

georges.triantafillidis@culture.gouv.fr

RÉSUMÉ

Un don important fait au Musée Archéologique de Strasbourg, constitué de la documentation scientifique et de mobilier archéologique issu des recherches d'Arthur Stieber (archéologue alsacien, 1908-1985), est à l'origine d'un projet collectif de recherche coordonné par le musée archéologique et la DRAC Alsace. Grâce à la richesse de la documentation recueillie (environ 500 carnets de fouille, des photographies, des plans et dessins...), ce projet se propose, selon trois axes, de redécouvrir la personnalité $d^{\prime} A$. Stieber et ses méthodes de travail, de faire une étude du fonds en tant qu'objet patrimonial et archivistique et enfin, de réaliser une analyse scientifique des données.

À travers l'exemple de ce projet, I'article montre l'intérêt de l'exploitation d'un fonds

Mots-CLÉs

Arthur Stieber,

projet collectif de recherche, archives scientifiques,

archives de l'archéologie,

documentation archéologique,

carnet de fouille,

valorisation,

musée archéologique de

Strasbourg,

DRAC Alsace.
A significant scientific documentation and archaeological material donation made by Arthur Stieber's descendants (Alsatian archaeologist, 1908-1985) to the Archaeological Museum of Strasbourg allowed to start a collective research project coordinated both by the Archaeological Museum and DRAC Alsace. Thanks to the wealth of the material collected (about 500 field notebooks; photographs, plans and drawings ...), this project proposes three axes of study:

- Stieber's personality and methods of work ;

- archival description, digitalization ;

- scientific analysis.

Through this project, the article shows specific interests of research archives collections and deals with their patrimonial enhancement issues.
Keywords

Arthur Stieber, collaborative research project, scientific records, archaeological archives, archaeological documentation, field notebook (book search), development (Valuation, Promotion),

Archaeological Museum of Strasbourg, DRAC Alsace. 


\section{ARTHUR STIEBER (1908-1985) : DE LA PROSPECTION DE TERRAIN À LA MISE EN CEUVRE DE LA CARTE ARCHÉOLOGIQUE}

Arthur Stieber [1] est né le 28 mars 1908 dans une famille d'agriculteurs de Furdenheim dans le Kochersberg, en Alsace. Il restera toute sa vie très attaché à ce riche terroir agricole où il commence ses premières prospections dès l'adolescence. Son intérêt pour l'archéologie débuterait dans les années 1920 et se poursuivrait sans interruption notable jusqu'en 1982, d'après les dates mentionnées dans ses carnets de terrain.

Il obtient un diplôme d'ingénieur-chimiste en 1929, puis soutient une thèse de doctorat en sciences à Paris en novembre 1933. Sa seconde thèse, "Mesure de la température de fusion des éléments très réfractaires $»$, fondée sur le rayonnement, sera mise à profit ultérieurement pour l'élaboration d'une méthode de prospection archéologique. En 1936, il s'inscrit aussi en thèse de doctorat en Lettres à I'Université de Strasbourg en prenant comme thème de recherche l'occupation du sol sur les terrasses de lœss dans la région du Kochersberg à l'époque néolithique.

Figure 1

Carte de visite d'Arthur Stieber (cliché DRAC Alsace)

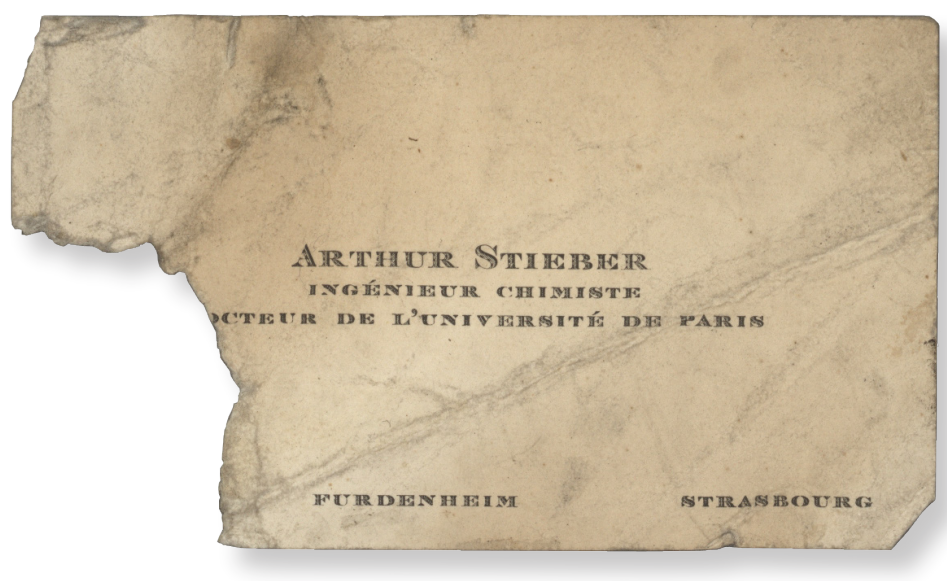

En octobre 1950, il entre comme stagiaire au CNRS sous la direction de Paul Wernert, directeur des Antiquités préhistoriques d'Alsace, avec pour mission d'assurer la surveillance archéologique des travaux d'aménagement (en particulier lors des travaux d'installation de réseaux d'assainissement et d'adduction d'eau potable dans les communes du Bas-Rhin). Il continue également ses prospections dans le Kochersberg. Ce prodigieux travail, mené systématiquement durant de longues années et complété par le dépouillement des sources documentaires, lui permet de localiser plus d'un millier de nouveaux emplacements archéologiques datables de la Préhistoire aux Temps modernes.

Devenu attaché de recherche au CNRS, il y mène toute sa carrière d'octobre 1952 à mars 1973. Dans ce cadre, il est chargé plus particulièrement de l'établissement d'un fichier général des découvertes faites dans le Bas-Rhin, travail qui doit aboutir à une carte archéologique pour ce département. A. Stieber est aussi l'auteur d'une méthode de prospection particulière, la « pédophysique » issue de ses connaissances de chimiste et fondée sur le rayonnement du sol. Grâce à diverses subventions du CNRS, il se dote d'un matériel de plus en plus performant qu'il va expérimenter, avec plus ou moins de succès, tout au long de ses prospections de terrain.

Après son décès en 1985, sa collection et ses archives restent conservées pendant une quinzaine d'années dans sa famille. C'est en 1999 que l'ensemble de cette vaste documentation scientifique et du mobilier archéologique associé fait l'objet d'un important don de la fille d'A. Stieber, Madame Vogel, au Musée archéologique de Strasbourg. Les archives documentaires (carnets, plans, relevés, photographies...) ont été remises par le musée au Service régional de l'archéologie (SRA) d'Alsace, afin que les innombrables informations inédites qu'elles contiennent puissent être exploitées dans le cadre national de la carte archéologique.

[1] SCHNitZLER 1985 


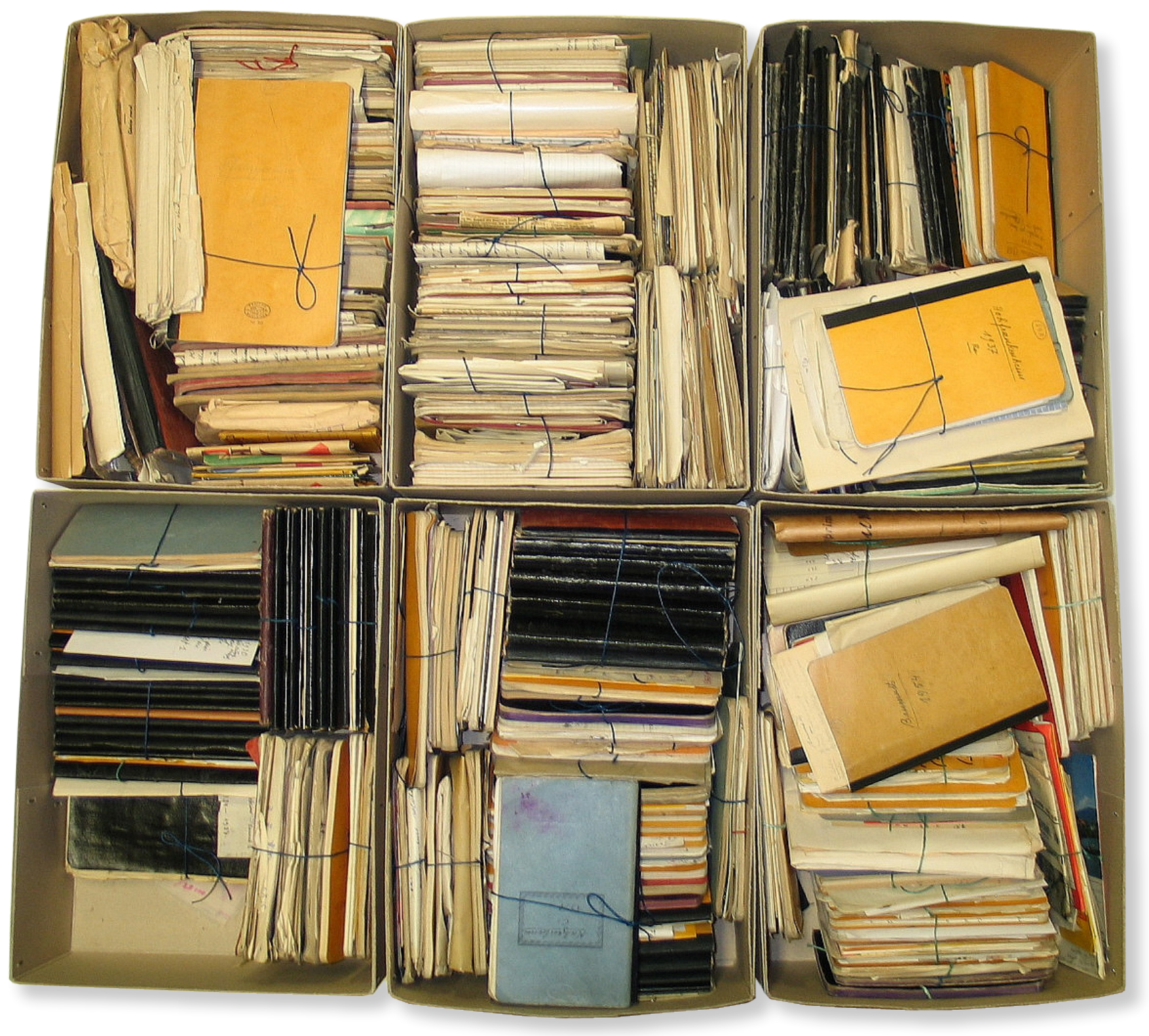

Figure 2

Carnets du fonds Stieber déposé à la DRAC Alsace (cliché DRAC Alsace)

\section{LA NAISSANCE D'UN PROJET COLLECTIF DE RECHERCHE}

Ce projet est né de la volonté de deux institutions, le Musée archéologique de Strasbourg et la DRAC Alsace, qui ont souhaité associer leurs compétences afin de traiter et valoriser le fonds Arthur Stieber au vu de ses multiples intérêts scientifiques. Le plus souvent, ce type d'archives est dispersé au gré de la carrière de l'archéologue, ou encore de sa vie personnelle, ce qui n'est pas le cas de ce fonds. Les carnets et les documents afférents constituent un ensemble très complet et homogène et ce sur près de soixante années de prospections archéologiques en Alsace. Par ailleurs, dépassant le cadre strict de la région, ce projet collectif de recherche s'inscrit dans un contexte plus large, à savoir la conservation des archives de l'archéologie. Une prise de conscience relative au devenir de la documentation de terrain s'est fait jour de manière simultanée dans les Ministères de la culture, et de l'enseignement supérieur et de la recherche, à la fin des années 1980. Depuis une dizaine d'années, cette question est centrale et de nombreux fonds du territoire national sont en cours d'inventaire. Le dépouillement des archives d'A. Stieber s'intègre dans ce processus de gestion et de valorisation de la documentation scientifique et participe au travail de mémoire de la discipline archéologique.

Le projet collectif de recherche a fait l'objet d'une année probatoire en 2013 et se poursuivra vraisemblablement jusqu'en 2017. Précisons que les résultats présentés 


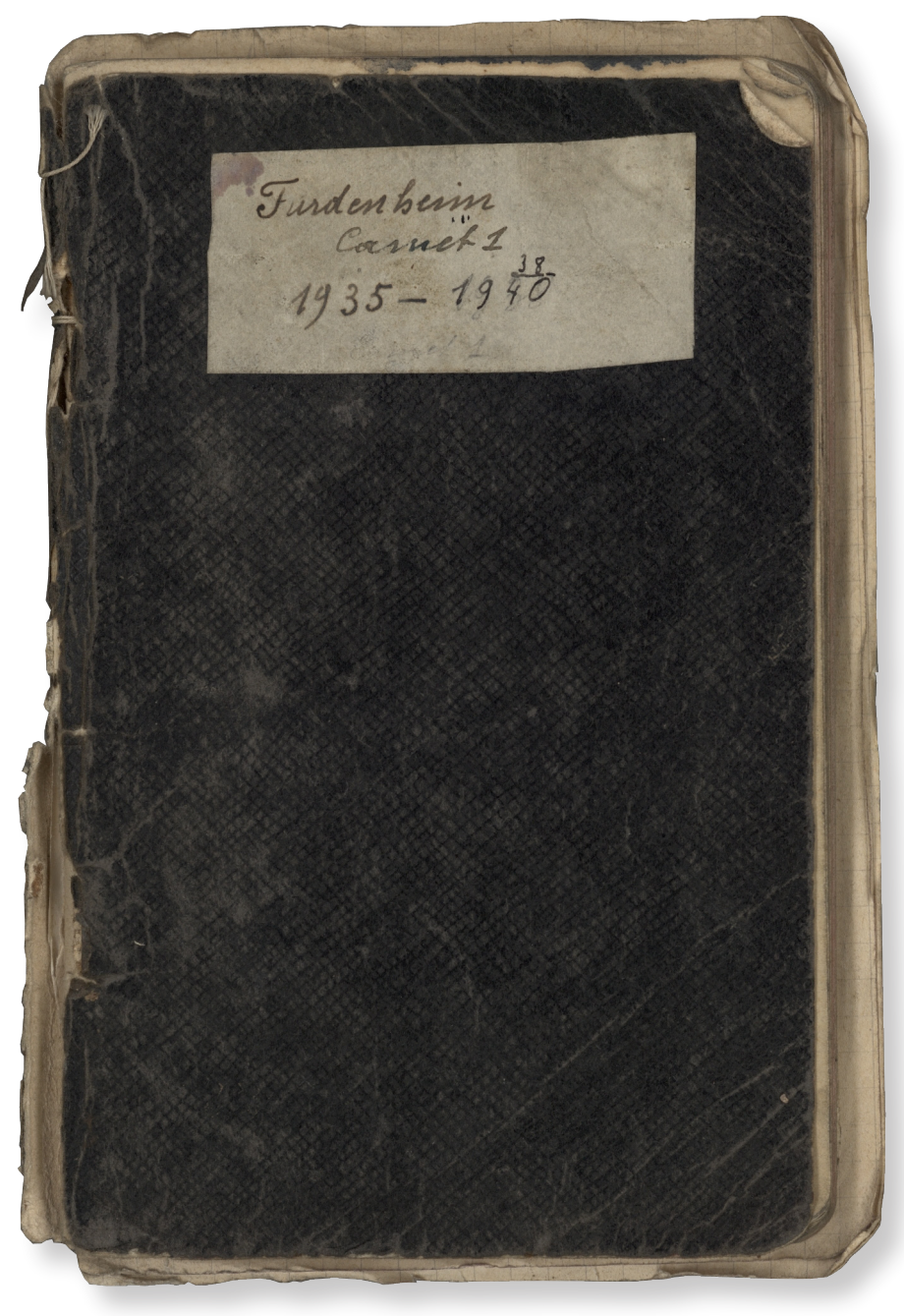

dans cet article sont issus de cette année probatoire et d'une partie de l'année 2014, soit moins d'un an et demi de recherches. Ils ne reflètent donc qu'une première analyse partielle du fonds. Par conséquent, à ce stade, nous ne dresserons qu'un état des lieux et ne tirerons pas de synthèse générale. Les jalons posés et les pistes de réflexion soulevées nécessiteront d'être vérifiés, affinés voire réorientés au fur et à mesure de l'étude pluri-annuelle. Des recherches complémentaires devront encore être effectuées pour étayer les hypothèses présentées dans le cadre de cette contribution.

\section{LE FONDS ARTHUR STIEBER}

Dans un premier temps a été réalisé un inventaire sommaire de l'ensemble des archives déposées au SRA, ce qui a permis d'appréhender celles-ci dans leur globalité. Le fonds $A$. Stieber se révèle être un ensemble parfaitement cohérent de documents de formes multiples. Il comporte en effet 506 carnets ou notes prises sur le terrain, dont $90 \%$ couvrent le

\section{Figure 3}

Carnet 1, Furdenheim, 1935-1940 (cliché DRAC Alsace)

Figure 4

Carnets ficelés par commune

(cliché Cécile Courtaud/DRAC Alsace)

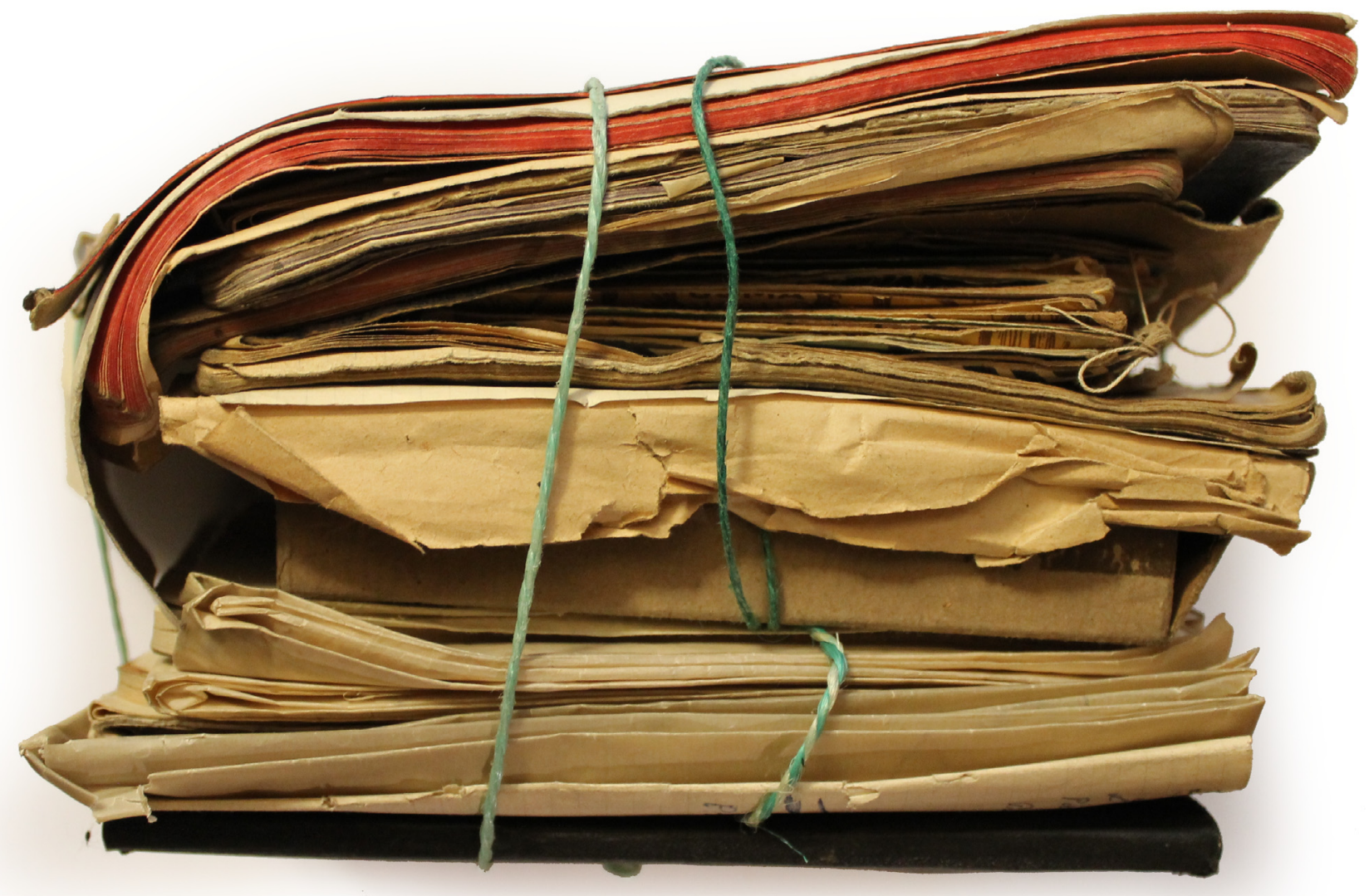


Bas-Rhin. Pour la plus grande majorité, les carnets sont de format quasi similaire $(17 \times 11 \mathrm{~cm}$ ou $17 \times 10 \mathrm{~cm})$. Quelques carnets parmi les plus anciens sont un peu plus grands $(21 \times 13 \mathrm{~cm}$ ou $21 \times 15 \mathrm{~cm})$. Tous les carnets présentent sur la couverture, le nom de la commune concernée, parfois une indication d'un lieu-dit ainsi qu'une ou plusieurs années. Un numéro à trois chiffres entouré, est également reporté sur un des carnets de chaque commune, mais pas de manière systématique. Il pourrait correspondre à une numérotation des communes selon un classement alphabétique des noms.

Chaque lot de communes a été lié par des ficelles par les soins d'A. Stieber. Des documents volants ont été joints à ces lots, il s'agit très souvent de notes prises sur des pages de carnet arrachées qui sont identifiées par la commune concernée et la date. La particularité de ces carnets est d'être accompagnés de nombreux documents collés à l'intérieur ou simplement intercalés entre les pages : des croquis, environ 80 articles de presse, des télégrammes, des brouillons d'articles en préparation, des notes, de la correspondance, 1690 tirages photographiques (et leurs négatifs), 34 plaques de verre et 2 plaques en métal gravé. À cela s'ajoute environ 250 cartes communales pour la plupart antérieures au remembrement du début des années soixante ainsi qu'un nombre encore indéterminé de documents produits et annotés de la main d'A. Stieber: dessins de mobilier, coupes, plans de localisation, calques d'après les plans cadastraux qui ont été conservés en rouleaux, par communes et par sites archéologiques.

Cette très riche documentation est le parfait reflet de l'intense activité d'A. Stieber, activité que I'on peut cerner également au travers des rapports annuels adressés au CNRS : «En somme, pendant cette année 1956, j'ai fait plus de 300 sorties sur le terrain, et repéré une soixantaine de points archéologiques nouvellement découverts, allant de l'époque néolithique aux temps mérovingiens et plus récents encore. »[2]

[2] Rapport adressé au CNRS, 1956, conservé au Musée archéologique de Strasbourg.

Figure 5

Rouleaux de cartes et plans (cliché Cécile Courtaud/DRAC Alsace)
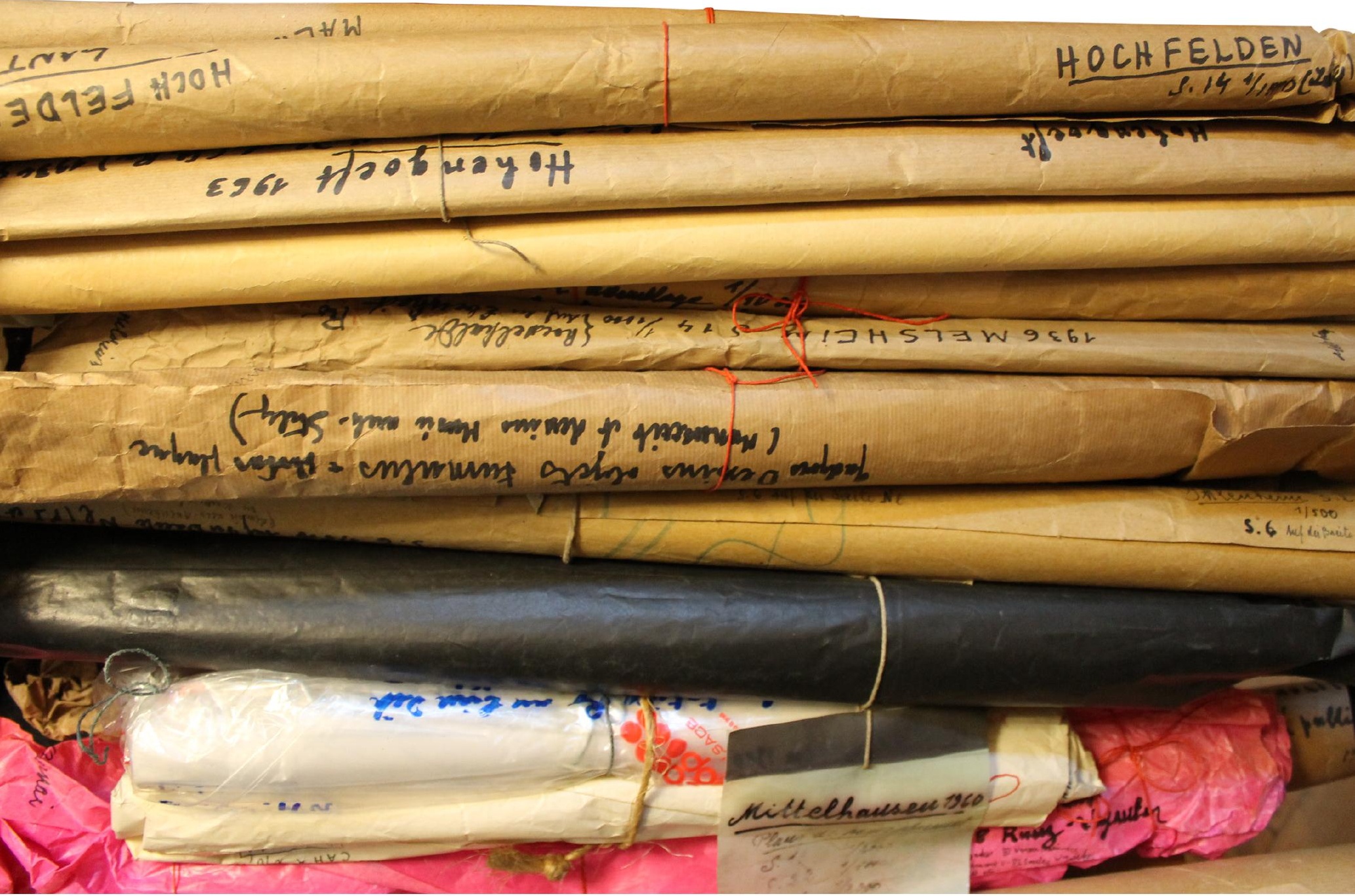


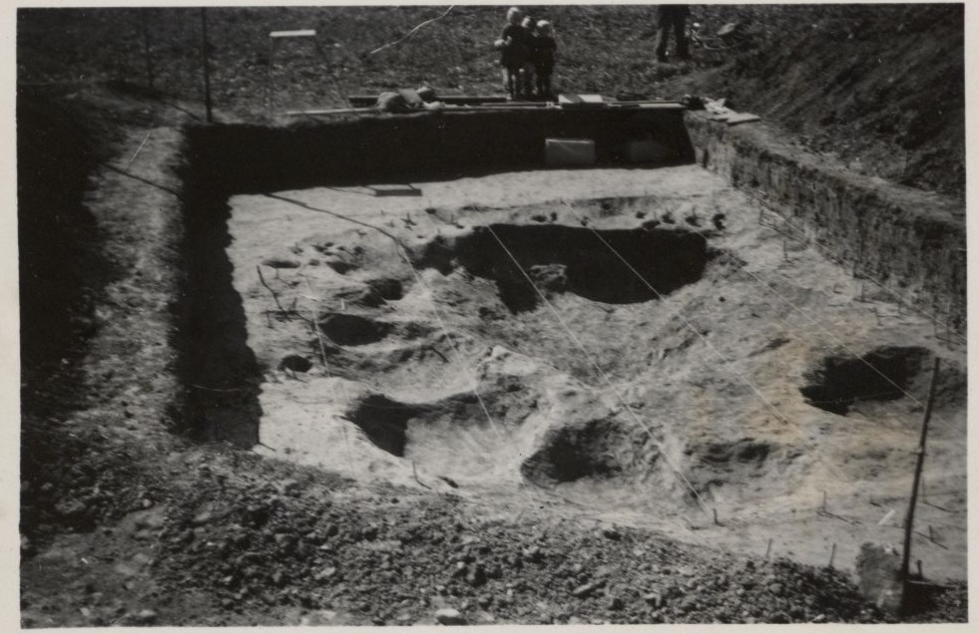

Figure 6

Carroyage lors d'une fouille à Furdenheim, «fond de cabane» Néolithique, 1946 (cliché DRAC Alsace)

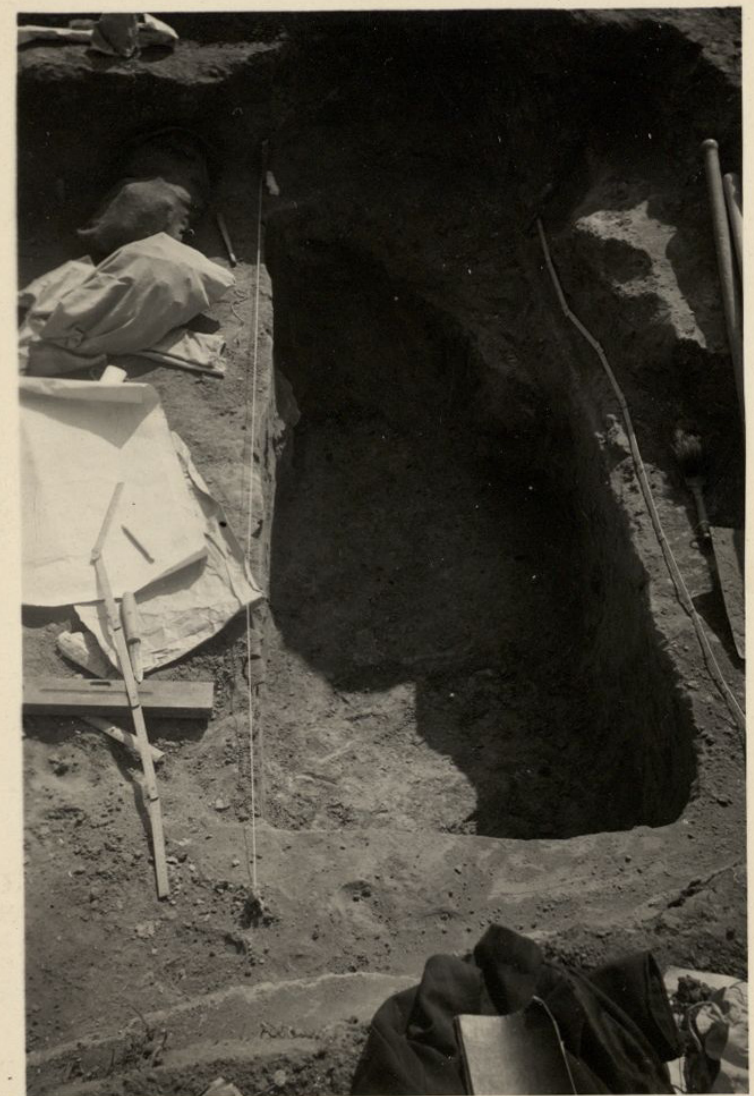

Fouille à Ittenheim, 1930 (cliché DRAC Alsace)

Figure 7

AVAIL

Considérant la richesse du fonds et les différentes pistes qui se profilaient, trois axes d'étude ont été dégagés très rapidement. Le premier axe a pour objet de redécouvrir le travail et la personnalité d'Arthur Stieber et de montrer la singularité de sa méthode archéologique. Le second axe consiste en l'étude du fonds en tant qu'objet patrimonial et archivistique en réalisant une description détaillée et une étude précise des carnets. Enfin, le troisième axe est une analyse scientifique des données en les comparant et les complétant avec celles déjà existantes et exploitables via une base de données. Ces nouvelles informations sont mises en perspective notamment avec celles constituant la carte archéologique.

Il convient de souligner ici le caractère transversal et pluridisciplinaire du projet et, de fait, la nécessité d'un travail collaboratif entre plusieurs institutions et partenaires. Ainsi aux deux institutions porteuses du projet, viennent

[3] Antéa-Archéologie, I'INRAP et le PAIR (Pôle d'archéologie interdépartemental rhénan).

[4] Le choix s'est porté sur Furdenheim qui est la commune de naissance $d^{\prime} A$. Stieber et qui est significative en terme de volume documentaire (16 carnets). Par ailleurs, cette commune fait partie du périmètre d'un précédent projet collectif de recherche sur l'occupation du sol dans la vallée de la Bruche (TriantAFILLIDIS \& OsWALD 2012). Les objectifs de ce projet étaient de comprendre la dynamique de l'occupation du sol par I'homme et le rapport entre I'homme s'ajouter des collaborateurs issus des opérateurs archéologiques œuvrant dans la région [3], de I'Université de Strasbourg, ou bien encore des sociétés savantes locales. Les trois axes de recherche développés sont interconnectés et les informations fournies par le fonds d'archives recomposeront peu à peu le puzzle de la vie et du travail d'A. Stieber. À plus large échelle, cette étude permettra d'appréhender I'archéologie alsacienne et la vie quotidienne au cours du $\mathrm{xx}^{\mathrm{e}}$ siècle, de compléter les sources publiées de l'archéologue et de fournir des inédits lors de l'exploitation scientifique des données.

Au cours de l'année 2013, une commune test [4] a été sélectionnée dans le but d'expérimenter une méthodologie de description archivistique et d'étude du fonds. À l'issue de cette année probatoire et face à la masse d'informations à traiter, trois secteurs géographiques ont été retenus selon une combinaison de critères [5] :

et I'environnement. Les sites de ce secteur ont été revisités et identifiés ces dernières années et le matériel afférent a été inventorié.

[5] Nos critères ont été définis à partir des observations faites sur la commune de Furdenheim. Ces critères combinent des communes représentatives en nombre de carnets, la présence de carnets antérieurs à 1945 (car il semble que ces données n'ont pas fait l'objet de publications) et des communes dont la localisation géographique correspond à différents milieux naturels. 
- zone humide, le secteur de Benfeld-Erstein (18 communes et 45 carnets) ;

- terres lœssiques, le secteur de Furdenheim-Pfettisheim

(24 communes et 179 carnets);

- collines sous-vosgiennes, le secteur de Willgottheim-

Hochfelden (23 communes et 40 carnets).

Les carnets et documents se rapportant à ces trois secteurs font l'objet d'une numérisation [6] et d'une description archivistique selon les normes en vigueur, dont le format EAD (Encoded archival description) en langage $\mathrm{XML}$, standard largement répandu dans le domaine des archives scientifiques. Les champs descriptifs et les thésaurus ont été sélectionnés de manière à être compatibles avec les bases de données nationales existantes (base Mémoire, etc.). Un travail de transcription de certains documents manuscrits a également été entamé afin d'en améliorer la lecture. Les méthodes d'enregistrement et de transcription employées reposent sur deux tableurs dont les données seront converties en XML EAD puis visualisées dans une application dédiée.

Le mode de restitution et d'exploitation numérique du projet et les moyens de valorisation du fonds sont encore en cours de réflexion [7].

\section{CE QUE RÉVÈLENT LES PREMIERS RÉSULTATS DU PROJET}

Au terme d'une année, la documentation relative à plus d'un tiers des communes retenues a été numérisée et un peu moins a été étudiée. Les documents ont révélé les méthodes de travail d'A. Stieber qui s'appuyait sur un réseau de connaissances locales le contactant notamment à chaque découverte fortuite.

Quelques pages de carnets abordent sa méthode de prospection -mesures, couleur du sol, etc. Ces carnets semblent constituer la mémoire écrite de l'archéologue, et une matière dans laquelle il va puiser les informations nécessaires à ses rapports ou ses publications (y sont insérés

[6] Chaque carnet et chaque document fait l'objet d'une version de conservation en tiff et d'une version de consultation en jpg. Le nommage des fichiers se veut le plus complet possible pour que l'intégrité du carnet et la place de tous les documents annexes soient respectés et lisibles.

[7] Cette partie du projet fait I'objet d'une collaboration mise en place avec I'IUT Robert Schuman d'Illkirch et des élèves de la licence professionnelle Médiation de I'information et du document dans les environnements numériques (Magali Jacquinez, Maryline Kreider, Jade Simonet), durant l'année scolaire 2014-2015.
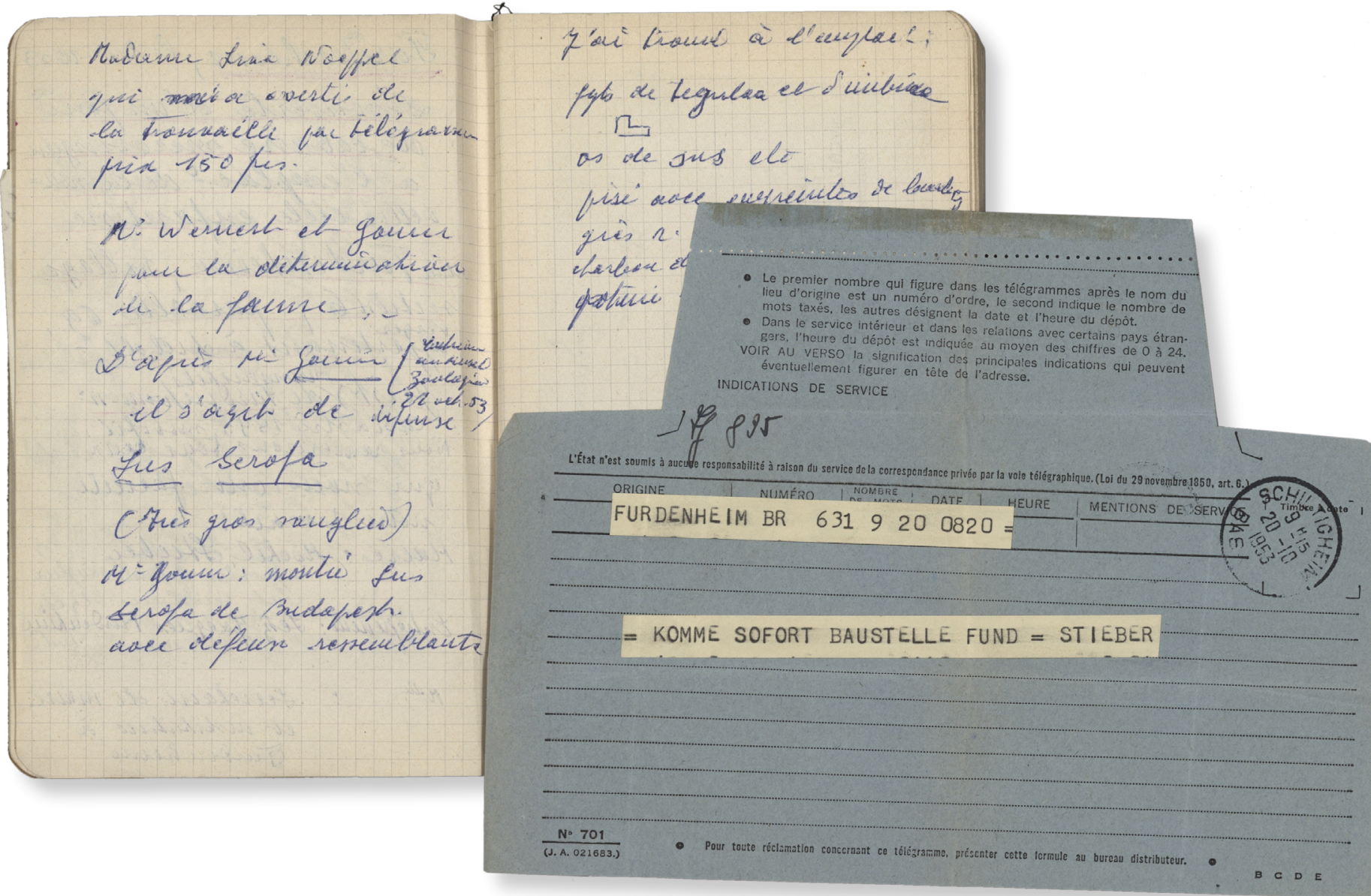


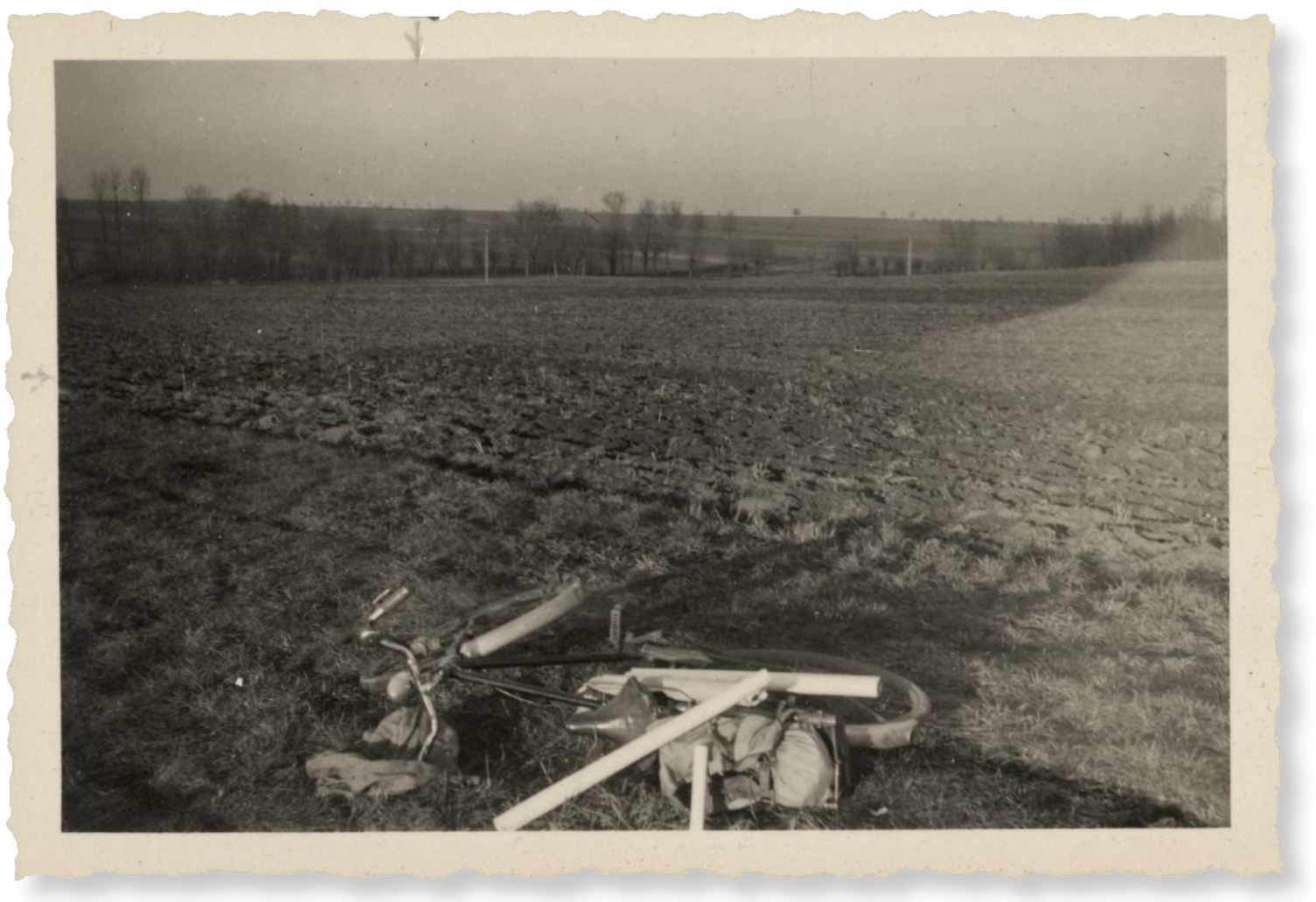

Figure 9

Vélo d'Arthur Stieber lors d'une prospection à Ittenheim, 1949 (cliché DRAC Alsace)

de nombreux brouillons d'articles, des références bibliographiques, des inventaires d'objets du Musée préhistorique et gallo-romain de Strasbourg - l'actuel Musée archéologique - ou encore des remerciements adressés à des personnes l'ayant aidé dans son travail). Toute découverte mentionnée par A. Stieber est scrupuleusement datée et référencée. L'archéologue note systématiquement la section du terrain, le lieu-dit, la parcelle, le nom du propriétaire et celui du locataire le cas échéant. Puis des croquis de plans de situation et des structures, des coupes, tous légendés et dimensionnés viennent préciser les découvertes. Enfin, il fait état de ses observations et des hypothèses qu'il en retire. Il prend soin de donner un maximum d'informations sur la nature des couches géologiques et il renseigne également les prises de vues réalisées en notant les conditions climatiques, la distance par rapport au sujet, les caractéristiques de I'appareil photographique et de la pellicule. Sa prise de note n'est pas strictement linéaire. Des passages écrits au crayon à papier ont été réécrits au stylo, des notes ont pu être complétées a posteriori, à la lumière de trouvailles plus récentes. Certains carnets regroupent des notes rédigées sur plusieurs années distantes ou ont été utilisés tête-bêche.

Toutes ces informations prises isolément peuvent paraître anecdotiques, mais une fois rassemblées et analysées, elles permettent de brosser le quotidien de l'archéologue, pas à pas.

L'étude des carnets mais aussi des documents annexes sont autant de traces du parcours professionnel de Stieber.
Des sources complémentaires au fonds ont également pu être repérées. Elles sont conservées dans d'autres structures telles I'Institut de France, le Museum national $d$ 'histoire naturelle ou encore les Archives du CNRS [8]. Il s'agit de quelques éléments de correspondance dans les papiers de Raymond Lantier [9] et de I'abbé Henri Breuil [10], tous deux préhistoriens, ainsi que du dossier de carrière d'Arthur Stieber contenant des documents administratifs, des rapports, des tirés-à-part, des photographies, etc. R. Lantier était en effet le «parrain» d'A. Stieber lors de son entrée au CNRS. A. Stieber a été en contact avec I'abbé Breuil et I'abbé Glory, mais la nature exacte de leurs échanges n'a pas encore pu être précisée à ce jour. Par ailleurs, il travaillait régulièrement avec Jean-Jacques Hatt, conservateur du Musée archéologique de Strasbourg et directeur régional des Antiquités. Enfin, de nombreuses mentions bibliographiques de Robert Forrer sont présentes dans les carnets de terrain.

D'un point de vue strictement documentaire, les carnets de Stieber sont de format standard, facilement transportables et ils rendent compte de la régularité des techniques

[8] Le dossier de carrière du personnel du CNRS sont versés aux Archives nationales (site de Fontainebleau).

[9] Archives de I'Institut de France, fonds Raymond Lantier, correspondance 1951 (Ms 8014), rapports et correspondance 1952-1956 (Ms 7989). R. Lantier a été nommé directeur de recherches d'A. Stieber en 1954.

[10] Bibliothèque du Museum d'histoire naturelle de Paris, fonds Breuil, correspondance 1961 ( $\mathrm{Br} 41)$. 


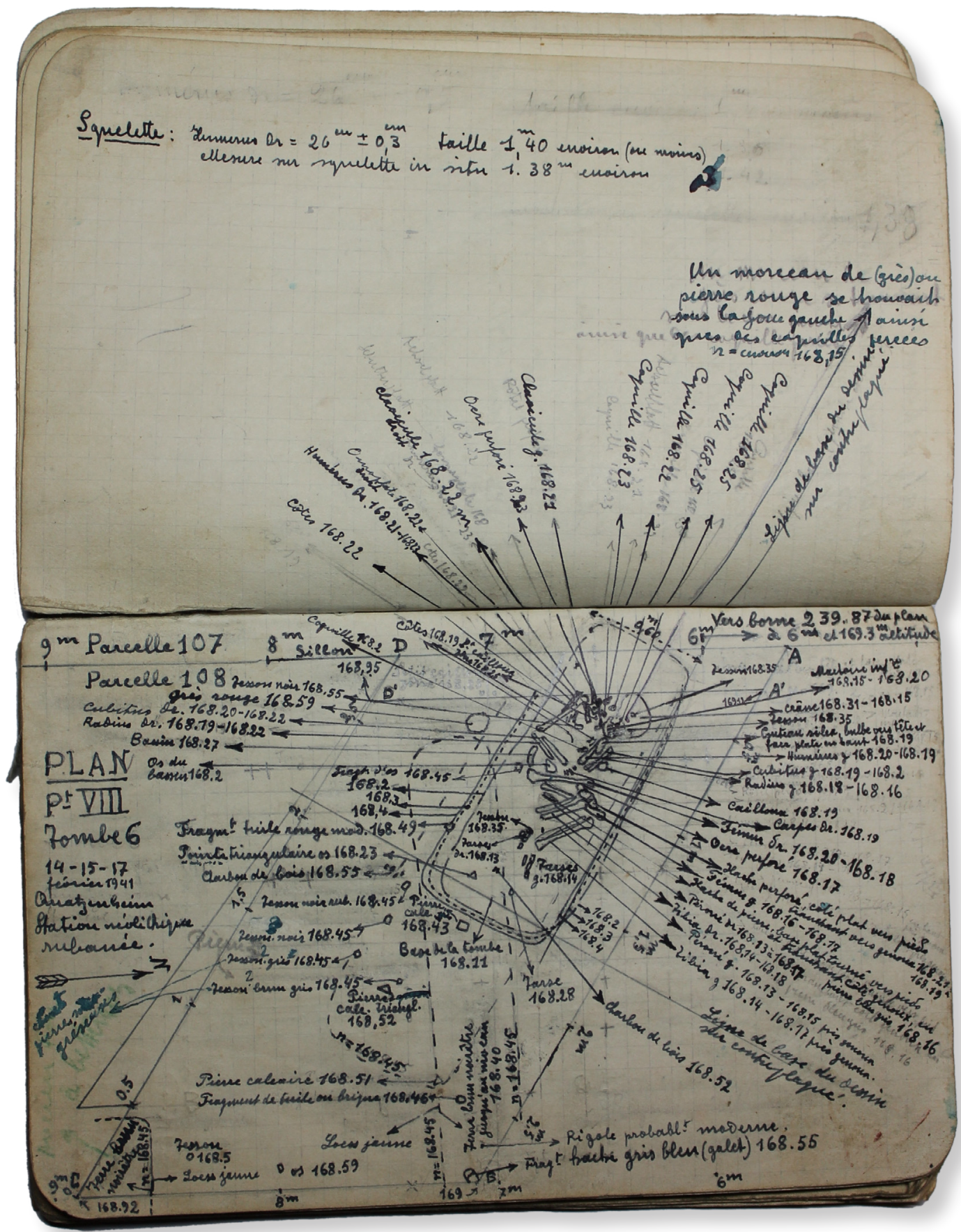

Figure 10

Découverte d'un squelette à Quatzenheim, 1940 (cliché DRAC Alsace)

d'enregistrement des informations. Mais lorsqu'on étudie de plus près tous les documents annexes, on en déduit que pour l'archéologue, tout est support pour une prise de notes : un prospectus, une carte de visite, un carton d'emballage alimentaire, un ticket de la compagnie des tramways strasbourgeois, etc. Une approche ethnologique du fonds A. Stieber, via le dépouillement des archives, pourrait être l'occasion de mettre au jour nombre d'informations qui reflètent la vie quotidienne de l'archéologue mais aussi celle de toute une population. En effet, au-delà du champ archéologique, les carnets nous renseignent sur d'autres domaines relatifs à l'histoire culturelle et sociale de I'Alsace. Ils contiennent parfois des indications démographiques sur les communes fouillées, des observations géomorphologiques ou sur les types de culture, ou encore la profondeur des labours. Des photographies de maisons ou de fermes prises dans les villages alsaciens témoignent de l'architecture traditionnelle de la région. Enfin, A. Stieber a réutilisé tout type de supports pour ses notes, jusqu'à des cartons d'emballages découpés qui nous révèlent un pan de I'histoire des entreprises locales dont certaines ont disparu depuis.

Par ailleurs, les carnets d'A. Stieber racontent en filigrane I'histoire de la discipline, par exemple en donnant un aperçu des conditions de travail au milieu du $x x^{e}$ siècle (relevé des noms, date de naissance, situation familiale des ouvriers employés sur les chantiers, ainsi que leurs salaires et horaires de travail selon leur fonction). 


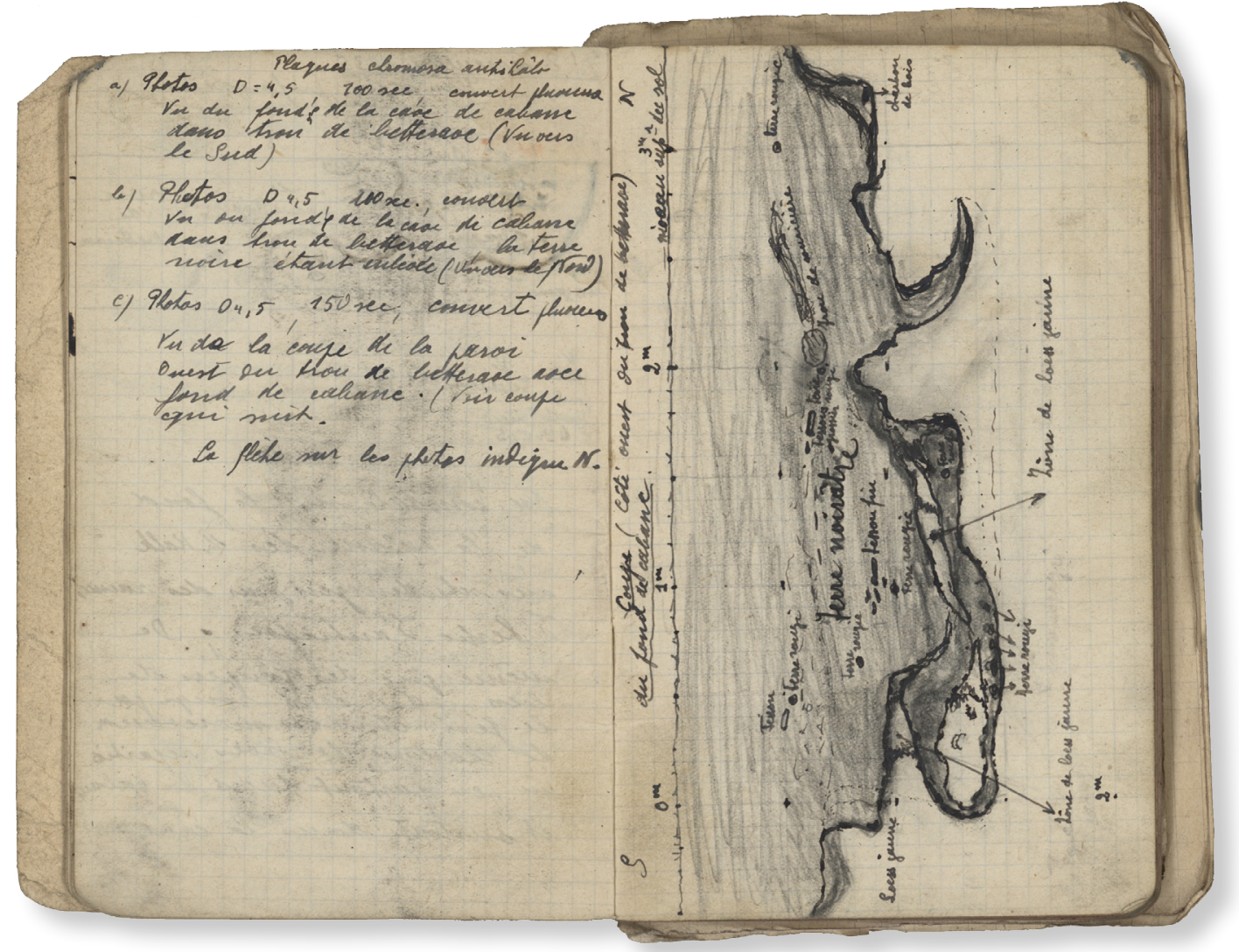

Figure 11

Coupe côté ouest du trou de betteraves, Furdenheim, 1935 (cliché DRAC Alsace)

Figure 12

Support de notes d'A. Stieber, cartons d'emballages des Établissements Ungemach (cliché DRAC Alsace)
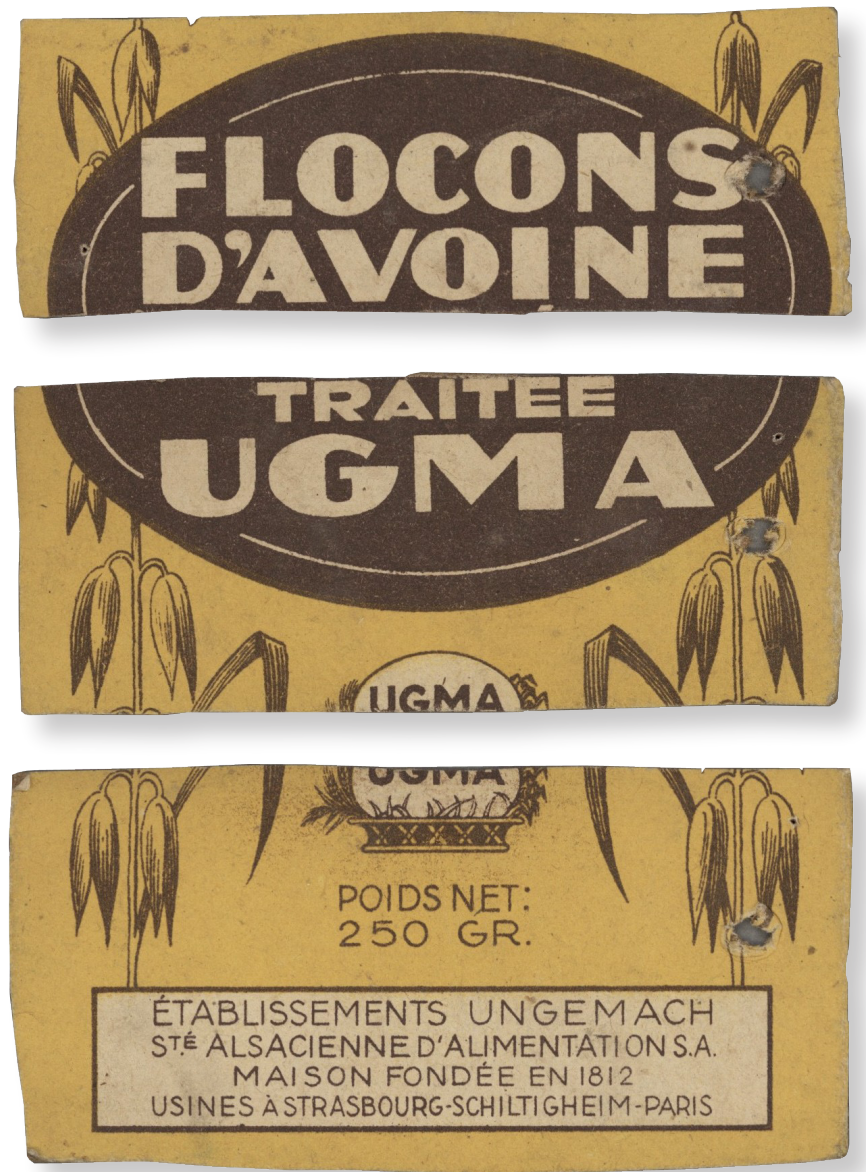

De précieuses informations sur les méthodes de travail, les techniques de fouille et les conditions sur le terrain sont distillées au fil des clichés photographiques amassés par l'archéologue au cours de sa longue carrière. Au vu de la masse d'informations collectée et de leur diversité, il paraît intéressant de développer plus avant cette approche ethnologique sous-jacente au projet. À propos de I'exploitation des données, I'objectif de travail durant cette première année consistait, d'une part, à évaluer et vérifier la fiabilité des informations contenues dans les carnets, en les croisant avec les données de la carte archéologique et avec les récents travaux scientifiques (fouilles, projets collectifs de recherche) et, d'autre part, à compléter la carte archéologique nationale. Cette démarche impliquait d'établir, dans un premier temps, un état de nos connaissances en s'attachant, plus particulièrement, à recenser les correspondances entre les données actuellement enregistrées dans la base Patriarche [11] et celles émanant des carnets (opérations archéologiques, entités archéologiques, bibliographie).

L'étude de ces carnets a démontré que presque tous les sites inédits proviennent des carnets antérieurs à 1945. Les observations datées d'après-guerre ont été

[11] Base nationale recensant l'ensemble des entités et opérations archéologiques sur le territoire métropolitain. Maintenue par le Ministère de la culture, elle est alimentée au niveau régional par chaque SRA. 


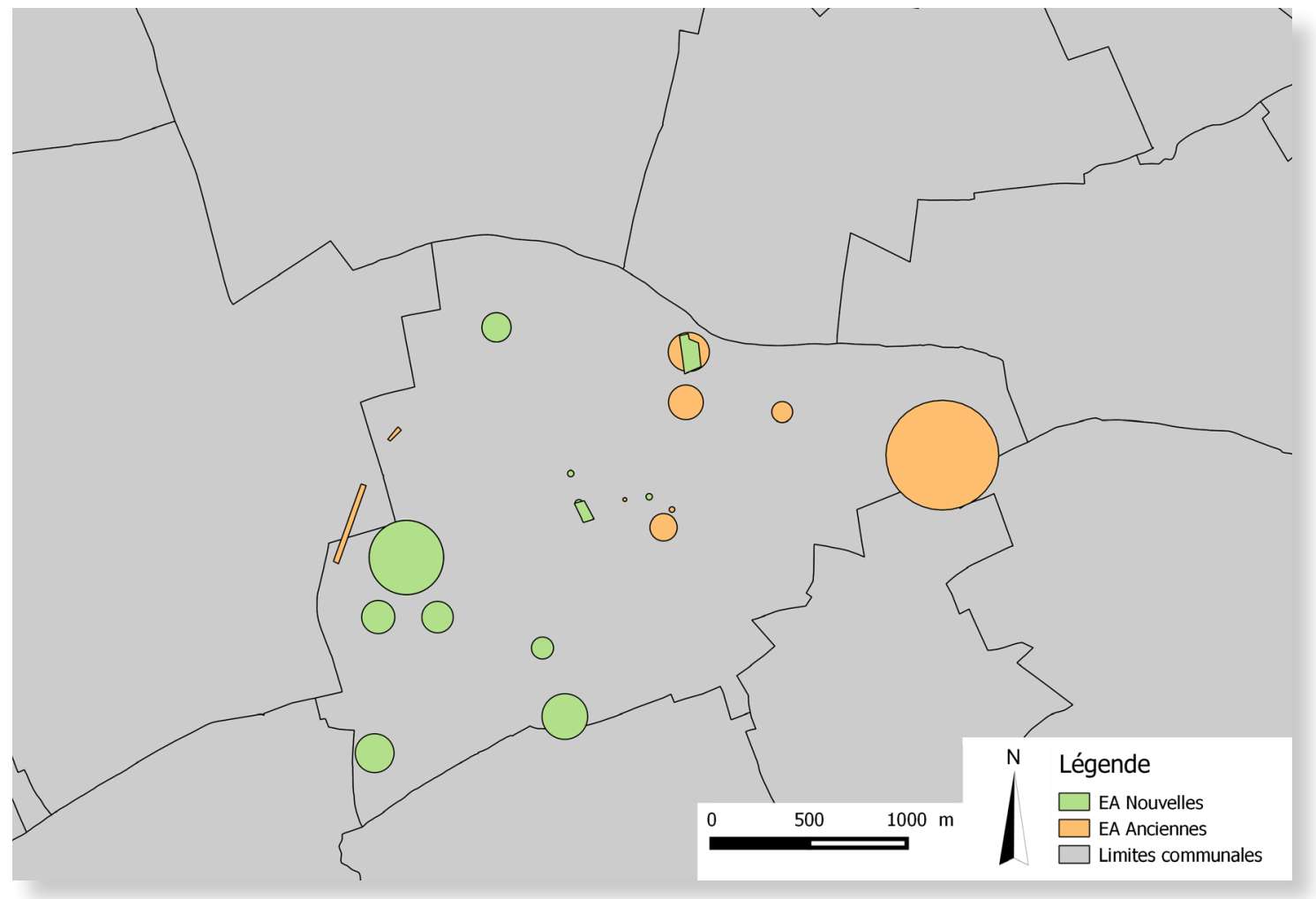

Figure 13

Carte des entités répertoriées à Furdenheim, avant et après l'étude des carnets Stieber (carte : Georges Triantafillidis/ DRAC Alsace) publiées et sont connues du SRA Alsace. Ces informations, enregistrées dans la base Patriarche, sont issues des observations $d^{\prime} A$. Stieber qui ont été transmises uniquement par ses articles, publiés essentiellement dans le Bulletin de la société préhistorique française (BSPF) et les Cahiers alsaciens d'archéologie, d'art et d'histoire (CAAAH).

En outre, les entités archéologiques, découvertes en grande partie lors de travaux agricoles, sont localisées essentiellement en milieu rural, et leur répartition chronologique s'étend du Néolithique à la période moderne. Les vestiges découverts concernent souvent des fosses et leur interprétation est parfois difficile, puisque les observations sont la plupart du temps lacunaires, en raison des circonstances de la découverte (labours ou travaux d'assainissement). En revanche, le dépouillement des carnets d'A. Stieber a permis d'inventorier 12 nouvelles entités uniquement pour la commune de Furdenheim, ce qui double le nombre d'entités archéologiques dans la base Patriarche.

\section{PERSPECTIVES}

À cette étape du projet, les perspectives de valorisation du fonds restent très ouvertes et plusieurs pistes sont à l'étude. Une fois numérisé et partiellement transcrit, le fonds sera consultable via un instrument de recherche intégré dans une application spécifique telle que Pleade. Cette base de données pourra être accessible chez les différents partenaires du projet dans un premier temps, en élargissant par la suite le partenariat vers d'autres institutions. Certaines questions restent encore en attente, à savoir : comment lier l'écrit et le mobilier conservé au Musée archéologique de Strasbourg ? Comment restituer au mieux la grande richesse de ce fonds ? Une exposition et une publication sont envisagées à l'issue de l'étude.

Au-delà du témoignage du travail de terrain d'A. Stieber, ce fonds présente un intérêt en tant qu'objet patrimonial, reflet de I'histoire de la discipline archéologique au cours du $x{ }^{e}$ siècle. Là encore, plusieurs axes se dessinent : de l'usage du carnet de terrain dans le travail de l'archéologue, jusqu'à une réflexion plus large sur l'utilisation du carnet comme support d'écriture transdisciplinaire (carnets d'artistes, de voyages, carnets d'observations scientifiques, etc.). Ces questions seront développées durant la dernière phase du projet. 


\section{BIBLIOGRAPHIE MENTIONNÉE}

Schnitzler, Bernadette, 1985, «En mémoire d'Arthur Stieber, (1908-1985) », Cahiers alsaciens d'archéologie, d'art et d'histoire 28, p. 125-126.

Schnitzler, Bernadette, Stahl, Marie \& Triantafillidis, Georges, 2013, Projet collectif de recherche : archives scientifiques de l'archéologie : fonds Arthur Stieber : année 2012, Strasbourg.

Schnitzler, Bernadette, Stahl, Marie \& Triantafillidis, Georges, 2014, Projet collectif de recherche : archives scientifiques de l'archéologie : fonds Arthur Stieber : année 2013, Strasbourg.

Schnitzler, Bernadette, Stahl, Marie \& Triantafillidis, Georges, 2015, Projet collectif de recherche : archives scientifiques de l'archéologie : fonds Arthur Stieber : année 2014, Strasbourg.

Triantafillidis, Georges \& Oswald, Grégory, 2012, Projet collectif de recherches : occupation du sol dans la vallée de la Bruche de la Préhistoire à l'Antiquité : rapport final, Strasbourg. 